\title{
A thorough study of the intriguing X-ray emission from the Cartwheel ring ${ }^{\star}$
}

\author{
A. Wolter and G. Trinchieri
}

\author{
Osservatorio Astronomico di Brera, via Brera 28, 20121 Milano, Italy \\ e-mail: anna@brera.mi.astro.it
}

Received 20 January 2004 / Accepted 30 June 2004

\begin{abstract}
We present the results from the high resolution Chandra observation of the Cartwheel galaxy. Many individual sources are resolved in the image, mostly associated with the outer ring. All detected sources have a very high X-ray luminosity $\left(\geq 10^{39} \mathrm{erg} \mathrm{s}^{-1}\right.$ ) that classifies them as Ultra Luminous X-ray sources (ULX). The brightest of them is possibly the most luminous individual non-nuclear source observed so far, with $L_{\mathrm{X}} \sim 10^{41} \mathrm{erg} \mathrm{s}^{-1}$ (at $D=122 \mathrm{Mpc}$ ). The spatial extent of this source is consistent with a point source at the Chandra resolution. The luminosity function of individual X-ray sources extends about an order of magnitude higher than previously reported in other galaxies. We discuss this in the context of the "universal" luminosity function for High Mass X-ray Binaries and we derive a Star Formation Rate higher than in other starburst galaxies studied so far. A diffuse component, associated with hot gas, is present. However, deeper observations that we will obtain with XMM-Newton are needed to constrain its properties.
\end{abstract}

Key words. galaxies: general - X-rays: galaxies - galaxies: luminosity function, mass function galaxies: individual: Cartwheel - galaxies: interactions

\section{Introduction}

The Cartwheel galaxy is a spectacular object, with the peculiar appearance reminiscent of a wheel (hence the name), most probably the result of an impact with one of the companion galaxies. It is located in a tight, compact group (SCG 0035-3357; Iovino et al. 2003) of 4 members, very close in space $\left(\sim 0.3 \mathrm{Mpc}^{1}\right.$ and velocity $\sim 400 \mathrm{~km} \mathrm{~s}^{-1}$ from one another, see Taylor \& Atherton 1984). Whether the impact was due to G3 (at $\sim 1^{\prime}$ NE, Higdon 1996) or G2 (at $\sim 3^{\prime}$ to the North; Athanassoula et al. 1997), two rings are now visible as a result: the outer one has the largest linear diameter measured in ring galaxies: $80^{\prime \prime}(\sim 100 \mathrm{kpc})$ along the major axis; the inner one, close to the core, is elliptical in shape with obvious dust lanes crossing it (Struck et al. 1996).

Many detailed observations of the Cartwheel are available, ranging from radio line (Higdon 1996) and continuum (Higdon 1996), to near- (Marcum et al. 1992) and far-infrared (Appleton \& Struck-Marcell 1987), optical (Theys \& Spiegel 1976; Fosbury \& Hawarden 1977) and $\mathrm{H} \alpha$ images (Higdon 1995) and line spectroscopy (Fosbury \& Hawarden 1977). All have confirmed the presence of a recent starburst in the outer

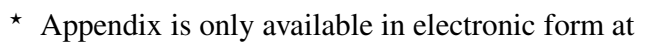
http://www.edpsciences.org

1 We use $H_{0}=75 \mathrm{~km} \mathrm{~s}^{-1} \mathrm{Mpc}^{-1}$, which implies a scale of $1.252 \mathrm{kpc} / \operatorname{arcsec}$ at the distance of the group, $D_{\mathrm{L}}=122 \mathrm{Mpc}$, throughout the paper.
}

ring, without a corresponding activity in the inner ring, nucleus or spokes, believed to be relatively devoid of gas. Most of the activity is confined in fact in the S-SW portion of the ring, where massive and luminous HII regions characterized by large $\mathrm{H} \alpha$ luminosities and equivalent widths are found (Higdon 1995). Both dynamical considerations and stellar evolution models suggest an age of $2-4 \times 10^{8} \mathrm{yr}$ for the star burst. The estimated supernova rate, as high as $1 \mathrm{SN} / \mathrm{yr}$ (i.e. almost two orders of magnitude higher than in normal galaxies), coupled with the evidence of a very low metallicity environment (as measured from $\mathrm{O}, \mathrm{N}$ and $\mathrm{Ne}$ ) also supports the view that star formation in the ring is a recent phenomenon and that the gas currently forming stars was nearly primordial at the time of the impact (Fosbury \& Hawarden 1977; Higdon 1995; Marcum et al. 1992).

We have imaged the Cartwheel for the first time in the X-ray band, using the HRI on board ROSAT (Wolter et al. 1999), and were able to attribute most of the emission to the outer ring, stronger in the Southern quadrant and very clumpy in nature. We therefore asked and obtained Chandra data with the ACIS-S in imaging mode, to better study the spatial distribution of the emission. We present here the Chandra data, and a discussion of the detection of both a diffuse component and a number of Ultra-Luminous X-ray sources well in excess of expectations.

The paper is organized as follows: in Sect. 2 we present the Chandra data; in Sect. 3 the results of the analysis for the 

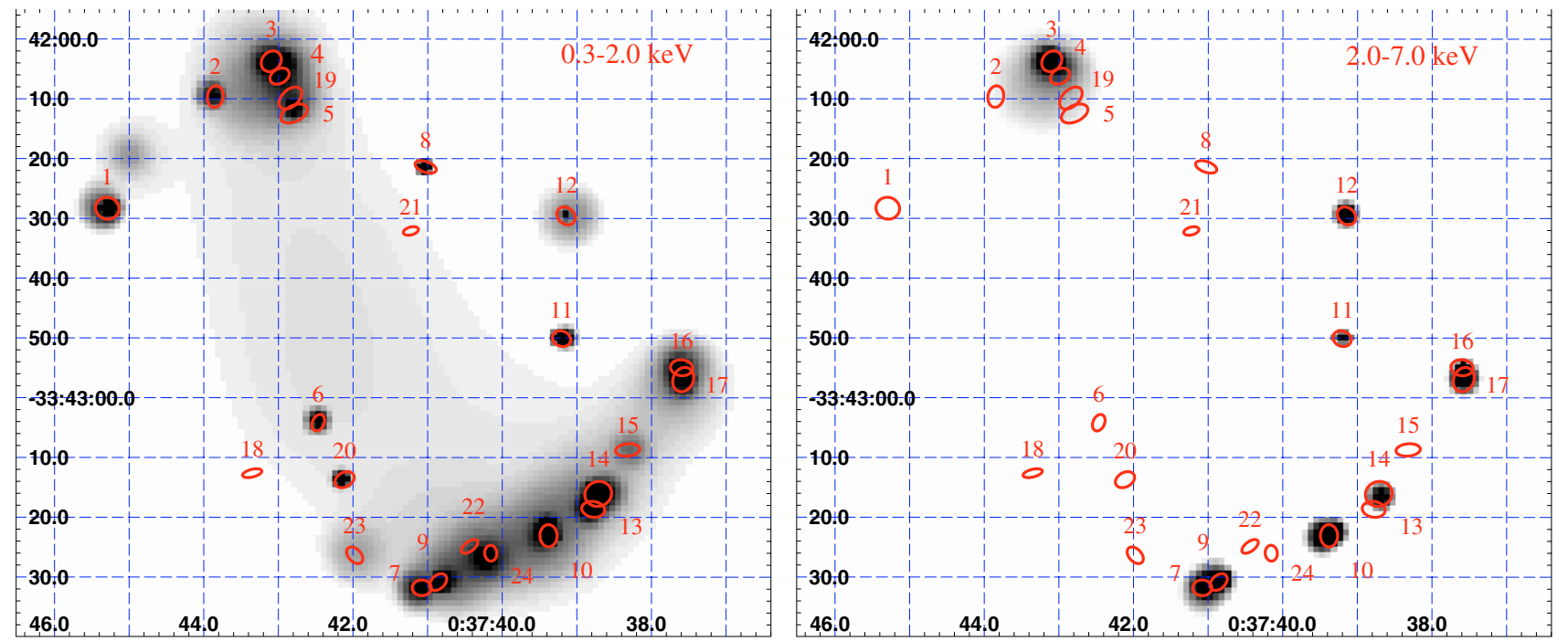

Fig. 1. Chandra image of the Cartwheel in the (left) soft $(0.3-2 \mathrm{keV})$ and (right) hard (2.0-7.0 keV) energy bands. An adaptive smoothing algorithm has been applied to the data (see text). Numbers identify sources as given in Table 1, with regions used to derive the source counts (from wavdetect).

individual sources and for the extended component; in Sect. 4 we discuss the results and present the X-ray Luminosity Function of the Cartwheel sources. Section 5 summarizes our findings.

\section{Chandra data}

The Cartwheel was observed by Chandra on 26-27th May 2001, with ACIS-S in imaging mode, operated in the standard full-frame mode, with an integration time of $3.2 \mathrm{~s}$. The backilluminated ACIS-S S3 chip was chosen for its soft response to detect the low temperature component. Telemetry was in faint mode. For a description of the Chandra mission see Weisskopf et al. (2000).

The data were reduced with the standard Chandra pipeline with the CIAO software (version 2.3) and the most recent available calibration products, as described in http://asc.harvard.edu/. The corrections applied are those appropriate for the ACIS-S instrument.

No evidence of in-orbit high background was found in the data, so the net exposure time is $76.1 \mathrm{ks}$.

Figure 1 shows the adaptively smoothed images of the Cartwheel in two energy bands $(0.3-2.0 \mathrm{keV}$ and $2.0-7.0 \mathrm{keV}$ respectively; the energy limits are chosen as a good compromise that maximizes the signal and minimizes the particle background contribution and calibration uncertainties). Both images show a very clumpy emission, mostly confined to the outer ring (see also Wolter et al. 1999; Wolter \& Trinchieri 2003; Gao et al. 2003). Individual sources that appear pointlike at the Chandra resolution $(\leq 1.5 \mathrm{kpc}$ at the Cartwheel distance, see Sect. 3.1.1) account for most of it, in particular in the harder energy band (right panel). A fraction of the counts ( $\sim 20 \%$ of the total) is found in a more extended component, that appears in the softer energy band. An additional component, of very low surface brightness, appears to connect the southern portion of the ring to the two nearby companions G1

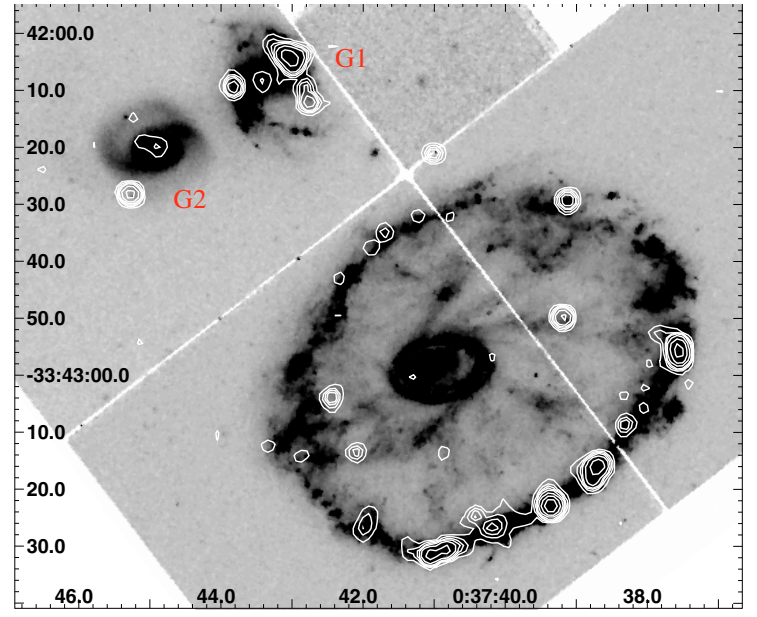

Fig. 2. The X-ray contours in the $0.3-7 \mathrm{keV}$ Chandra image over-plotted on the optical image in the $5450 \mathrm{~W}$ filter from the HST archives. X-ray contours are: $7.9 \times 10^{-06}, 1.6 \times 10^{-05}, 2.4 \times 10^{-05}$, $3.2 \times 10^{-05}, 5.3 \times 10^{-05}, 1.1 \times 10^{-04}, 2.1 \times 10^{-04}, 4.2 \times$ $10^{-04} \mathrm{cnts} / \mathrm{s} / \operatorname{arcsec}^{2}$.

and G2 in the soft image (left panel of Fig. 1). No excess emission is detected at the position of the optical nucleus.

The X-ray contours from the smoothed $0.3-7 \mathrm{keV}$ image are shown in Fig. 2 superposed on to the HST image ${ }^{2}$ and show the correspondence of the X-ray emission with the outer ring.

We will explore all the evidence more quantitatively in the following sections.

\footnotetext{
2 The F450W filter image was obtained from the HST WFPC2 association archive. The image is based on observations made with the NASA/ESA Hubble Space Telescope, obtained from the data archive at the Space Telescope Science Institute. STScI is operated by the Association of Universities for Research in Astronomy, Inc. under NASA contract NAS 5-26555.
} 
Table 1. List of sources that are detected in the area covered by the Cartwheel system and displayed in Fig. 1. Unabsorbed fluxes are computed in the $0.5-2 \mathrm{keV}$ and $2-10 \mathrm{keV}$ bands assuming the best fit model of the sum of point sources (see text for details). Unabsorbed, k-corrected luminosities in $\mathrm{erg} \mathrm{s}^{-1}$ are in the $2-10 \mathrm{keV}$ band. The sources are also reported in the Appendix in RA order for completeness; however, in the Appendix the fluxes are all computed under a different spectral hypothesis (see text). These sources are identified by their number in the last column of Table A.1 for easy reference.

\begin{tabular}{|c|c|c|c|c|c|c|c|}
\hline $\bar{N}$ & Position (J2000) & Net counts & $\begin{array}{r}\text { Count rate } \\
\times 10^{3} \mathrm{~s}\end{array}$ & $\begin{array}{r}F(0.5-2 \mathrm{keV}) \\
\mathrm{erg} \mathrm{cm}^{-2} \mathrm{~s}^{-1}\end{array}$ & $\begin{array}{r}F(2-10 \mathrm{keV}) \\
\mathrm{erg} \mathrm{cm}^{-2} \mathrm{~s}^{-1}\end{array}$ & $\begin{array}{r}\log L_{X} \\
2-10 \mathrm{keV}\end{array}$ & $\begin{array}{r}\text { Notes } \\
\# \text { as in Gao }\end{array}$ \\
\hline 1 & $00: 37: 45.294-33: 42: 28.31$ & $73.21 \pm 8.77$ & $0.961 \pm 0.115$ & $3.59 \times 10^{-15}$ & $2.94 \times 10^{-15}$ & 39.72 & $\mathrm{a} ; \# 30$ \\
\hline 2 & $00: 37: 43.847-33: 42: 09.63$ & $39.87 \pm 6.56$ & $524 \pm 0.086$ & $1.96 \times 10^{-15}$ & $1.60 \times 10^{-15}$ & 39.45 & $\mathrm{a} ; \mathrm{b} ; \# 29$ \\
\hline 3 & $00: 37: 43.117-33: 42: 03.96$ & $72.32 \pm 8.72$ & $0.950 \pm 0.115$ & $3.55 \times 10^{-15}$ & $2.90 \times 10^{-15}$ & 39.71 & $\mathrm{a} ; \mathrm{b} ; \# 24$ \\
\hline 4 & $00: 37: 43.006-33: 42: 05.96$ & $48.56 \pm 7.14$ & $0.638 \pm 0.094$ & $2.38 \times 10^{-15}$ & $1.95 \times 10^{-15}$ & 39.54 & $\mathrm{a} ; \mathrm{b} ; \# 25$ \\
\hline 5 & $00: 37: 42.791-33: 42: 12.46$ & $40.58 \pm 6.63$ & $0.533 \pm 0.087$ & $1.99 \times 10^{-15}$ & $1.63 \times 10^{-15}$ & 39.46 & $\mathrm{a} ; \mathrm{b} ; \# 22$ \\
\hline 6 & $00: 37: 42.466-33: 43: 04.18$ & $19.97 \pm 4.69$ & $0.262 \pm 0.062$ & $9.79 \times 10^{-16}$ & $8.02 \times 10^{-16}$ & 39.15 & $\# 21$ \\
\hline 7 & $00: 37: 41.078-33: 43: 31.80$ & $70.64 \pm 8.60$ & $0.928 \pm 0.113$ & $3.46 \times 10^{-15}$ & $2.84 \times 10^{-15}$ & 39.70 & \#17 \\
\hline 8 & $00: 37: 41.028-33: 42: 21.38$ & $21.23 \pm 4.80$ & $0.279 \pm 0.063$ & $1.04 \times 10^{-15}$ & $8.52 \times 10^{-16}$ & 39.18 & $\mathrm{a} ; \# 16$ \\
\hline 9 & $.859-$ & 66.20 & $0.869 \pm 0.109$ & $3.25 \times 10^{-15}$ & $2.66 \times 10^{-15}$ & 39.67 & $\# 15$ \\
\hline 10 & $3: 43: 23.08$ & $83.77 \pm$ & $5.040 \pm 0.260$ & $1.88 \times 10^{-14}$ & $1.54 \times 10$ & 40.44 & \#11 \\
\hline 11 & $00: 37: 39.206-33: 42: 50.11$ & $62.59 \pm 8.06$ & $0.822 \pm 0.106$ & $3.07 \times 10^{-15}$ & $2.51 \times 10^{-15}$ & [39.65] & $\# 10$ \\
\hline 12 & $00: 37: 39.147-33: 42: 29.57$ & $43.75 \pm 6.78$ & $0.575 \pm 0.089$ & $2.15 \times 10^{-15}$ & $1.76 \times 10^{-15}$ & 39.49 & \#9 \\
\hline 13 & $00: 37: 38.781-33: 43: 18.66$ & $38.02 \pm 6.40$ & $0.499 \pm 0.084$ & $1.87 \times 10^{-15}$ & $1.53 \times 10^{-15}$ & 39.43 & \#7 \\
\hline 14 & 5.08 & .54 & $1.391 \pm 0$ & $5.19 \times 10^{-15}$ & $4.25 \times 10^{-15}$ & 39.88 & \#6 \\
\hline 15 & $00: 3$ & 16.06 & $0.211 \pm 0.056$ & $7.88 \times 10^{-16}$ & $6.45 \times 10^{-16}$ & 39.06 & \#5 \\
\hline 16 & $00: 37: 37.598-33: 42: 54.96$ & $94.51 \pm$ & $1.241 \pm 0.130$ & $4.64 \times 10^{-15}$ & $3.79 \times 10^{-15}$ & 39.83 & \#3 \\
\hline 17 & $00: 37: 37.576-33: 42: 56.94$ & $16.10 \pm 10.95$ & $1.525 \pm 0.144$ & $5.70 \times 10^{-15}$ & $4.66 \times 10^{-15}$ & 39.92 & $\# 2$ \\
\hline 18 & $3.351-33: 4$ & .83 & $0.090 \pm 0.037$ & $3.35 \times 10^{-16}$ & $2.74 \times 10^{-16}$ & 38.69 & $\mathrm{a}$ \\
\hline 19 & $00: 37: 42.841-33: 42: 09.63$ & 18.57 & $0.244 \pm 0.060$ & $9.11 \times 10^{-16}$ & $7.46 \times 10^{-16}$ & 39.12 & $\mathrm{a} ; \mathrm{b} ; \# 23$ \\
\hline 20 & $00: 37: 42.114-33: 43: 13.71$ & $13.20 \pm 3.87$ & $0.173 \pm 0.051$ & $6.47 \times 10^{-16}$ & $5.30 \times 10^{-16}$ & 38.97 & $\# 20$ \\
\hline 21 & $00: 37: 41.226-33: 42: 32.10$ & $6.97 \pm 2.83$ & $0.092 \pm 0.037$ & $3.41 \times 10^{-16}$ & $2.80 \times 10^{-16}$ & 38.70 & \\
\hline 22 & $00: 37: 40.438-33: 43: 24.87$ & $14.28 \pm 4.00$ & $0.188 \pm 0.053$ & $7.00 \times 10^{-16}$ & $5.73 \times 10^{-16}$ & [39.01] & \#13 \\
\hline 23 & $00: 37: 41.988-33: 4$ & & $0.112 \pm 0.042$ & $4.19 \times 10$ & $3.43 \times 10^{-16}$ & 38.78 & \#19 \\
\hline 24 & $00: 37: 40.154-33: 43: 26.00$ & $5.16 \pm 2.45$ & $0.068 \pm 0.032$ & $2.53 \times 10^{-16}$ & $2.07 \times 10^{-16}$ & 38.57 & $\# 12$ \\
\hline
\end{tabular}

N.B.: Sources \#4, \#8, \#18 and \#27 in Gao et al. (2003) are not detected by us. Our sources N.18 and N.21 are not detected by Gao et al. Luminosities in [ ] indicate sources that could be modulated by higher absorption because they appear to have a soft/hard count ratio different from the others.

a) Not used to compute the Luminosity Function.

b) Associated with G1; G2 is below our detection threshold.

\section{Analysis and results}

\subsection{Individual sources}

A wavdetect detection algorithm applied to the $0.3-7.0 \mathrm{keV}$ image provides 72 sources in the whole field. We have applied the algorithm using scales ranging from $1^{\prime \prime}$ to $1^{\prime}$ and a significance threshold of $10^{-6}$ that corresponds to 0.25 false sources in the image considered at any given scale. All detected sources are listed and discussed in the Appendix and in Table A.1.

In Table 1 we list the positions and count rates (in the $0.3-7.0 \mathrm{keV}$ band) of the 25 sources located in the Cartwheel region, most of which should be associated with it (see also Fig. 1 and Sect. 2).

By assuming the spectrum found for the sum of point sources (see Sect. 3.1.2), we compute the unabsorbed fluxes in the $0.5-2 \mathrm{keV}$ and in the $2-10 \mathrm{keV}$ bands. Assuming the distance of $122 \mathrm{Mpc}$ for the Cartwheel we list in Table 1 the logarithm of the k-corrected luminosity $L_{\mathrm{X}}$ in the $2-10 \mathrm{keV}$ band.

The brightest point source in the outer ring has a luminosity of at least $L_{\mathrm{X}} \sim 2-4 \times 10^{40} \mathrm{erg} \mathrm{s}^{-1}$ in the $0.5-2 \mathrm{keV}$ band,
Table 2. Spectral fit results for source N.10.

\begin{tabular}{lrr}
\hline \hline & \multicolumn{1}{c}{ Power law } & Multicolor disk \\
\hline$N_{\mathrm{H}} \times 10^{21} \mathrm{~cm}^{-2}$ & $3.6(2.6-5.9)$ & $2.4(0.6-3.4)$ \\
$\Gamma / k T$ & $1.6(1.3-2.0)$ & $1.3(1.0-2.1)$ \\
$\chi^{2}$ (d.o.f.) & $9.97(9)$ & $9.73(9)$ \\
& & \\
$F_{\mathrm{X}}(0.5-2 \mathrm{keV})$ obs. & $1.2 \times 10^{-14}$ & $1.2 \times 10^{-14}$ \\
$F_{\mathrm{X}}(0.5-2 \mathrm{keV})$ unabs. & $2.5 \times 10^{-14}$ & $1.8 \times 10^{-14}$ \\
$F_{\mathrm{X}}(2-10 \mathrm{keV})$ obs. & $5.0 \times 10^{-14}$ & $2.9 \times 10^{-14}$ \\
$F_{\mathrm{X}}(2-10 \mathrm{keV})$ unabs. & $5.2 \times 10^{-14}$ & $5.3 \times 10^{-14}$ \\
& & \\
$L_{\mathrm{x}}(0.5-2 \mathrm{keV})$ & $4.3 \times 10^{40}$ & $2.1 \times 10^{40}$ \\
$L_{\mathrm{x}}(2-10 \mathrm{keV})$ & $9.1 \times 10^{40}$ & $5.3 \times 10^{40}$ \\
$L_{\mathrm{x}}(0.05-100 \mathrm{keV})$ & $4.5 \times 10^{41}$ & $9 . \times 10^{40}$ \\
\hline
\end{tabular}

and of $L_{\mathrm{X}} \sim 5-9 \times 10^{40} \mathrm{erg} \mathrm{s}^{-1}$ in the $2-10 \mathrm{keV}$ band $^{3}$, one of the brightest individual sources ever found in galaxies. Even

${ }^{3}$ The luminosity depends on the spectral model assumed, see Table 2 . 
though at the Cartwheel distance the Chandra resolution defines a region of a few kpc that could contain more than one source, the high X-ray luminosity suggests either a single extremely bright source, or a very dense collection of several, high $L_{\mathrm{X}}$ sources, which is probably even more peculiar. A possible source variability suggested for this source (see Sect. 4.1) further suggests that this is indeed a single high $L_{X}$ source (or equivalently that a single source dominates the emission from this region).

No source is detected at the position of the optical nucleus of the Cartwheel, indicating that it is fainter than our detection limit of a few $\times 10^{38} \mathrm{erg} \mathrm{s}^{-1}$. An active nucleus is thus either not present or it is so heavily absorbed that even at $\sim 7 \mathrm{keV}$ no emission emerges.

A number of individual sources coincide with G1, for a total of 220 net counts, that correspond to a flux $f_{(0.5-2.0 \mathrm{keV})}=$

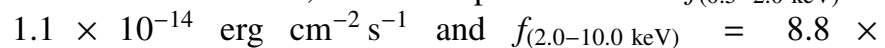
$10^{-15} \mathrm{erg} \mathrm{cm}^{-2} \mathrm{~s}^{-1}$; for a luminosity $L_{\mathrm{X}}=2.5 \times 10^{40} \mathrm{erg} \mathrm{s}^{-1}$ (0.5-10 keV band) (assuming the same spectrum of the sum of point sources, see Fig. 6 and Sect. 3.1.2). The disturbed optical morphology of G1 and the large number of discrete high $L_{\mathrm{X}}$ sources detected in the galaxy area are both consistent with an interaction scenario (G1 with G2, as suggested by Higdon 1996).

A source that might be positionally coincident with the nucleus is also detected at the location of G3 at $3.9 \sigma$ with a total of $9.6 \pm 3.3$ counts (see Appendix A). The corresponding flux obtained with the same spectrum as for G1 is $f_{(0.5-2.0 \mathrm{keV})}=4.6 \times 10^{-16} \mathrm{erg} \mathrm{cm}^{-2} \mathrm{~s}^{-1} ; f_{(2.0-10.0 \mathrm{keV})}=$ $3.9 \times 10^{-16} \mathrm{erg} \mathrm{cm}^{-2} \mathrm{~s}^{-1}$; for a total luminosity $L_{\mathrm{X}}=1.5 \times$ $10^{39} \mathrm{erg} \mathrm{s}^{-1}(0.5-10 \mathrm{keV}$ band).

While we were preparing this paper, a list of sources in the Cartwheel region was presented by Gao et al. (2003). We compare our list with that presented by them. Positions are generally consistent, however there is a small systematic shift of $\langle\Delta \mathrm{RA}\rangle=-0.25^{\prime \prime}$ in RA and $\langle\Delta \mathrm{Dec}\rangle=-0.60^{\prime \prime}$ in declination between our positions and those of Gao et al. A few sources are only present in either list (see notes to Table 1): it is likely that differences in the detection process and thresholds assumed cause the slightly different source lists; the effect is however limited to faint or confused sources. The count rates of the common sources are generally higher in the Gao et al. list. This might be the result of different bands used (the detection band is not indicated in the Gao et al. paper) and/or background subtraction considered and/or a different detection algorithm (under the IDL software).

\subsubsection{Extent}

In order to investigate the nature of the sources we have studied the radial profile of the brightest ones (i.e. with sufficient counts to perform a significant comparison with the Point Spread Function, PSF, obtained by ray tracing the photons distributed with the same spectral shape as the source under test with the CIAO task chart). The profiles of two of these sources are plotted in Fig. 3 in two different energy bands, after subtracting the detected nearby sources, together with the PSF binned in the same energy range.

The sources are unresolved at the resolution of the instrument, i.e. they have a core of $\lesssim 1^{\prime \prime}$. An extended source of lower surface brightness is present and it is stronger at soft energies for $r \geq 1^{\prime \prime}$. 5, most evident for source N.10. We infer that this is due to the underlying ring of star formation in the Cartwheel. The thickness of this component, $\sim 8-10^{\prime \prime}$, is comparable to that in the optical band.

We have also performed a number of projections, orthogonal to the ring, in a few spots of the southern ring. We plot two of these in Fig. 4. The location is given relative to the sources identified in Fig. 1. The $X$-axis is approximately along the radial distance from the center of the ring, from the inside towards the outside of the Cartwheel. The peak in the distribution corresponds to the ring, which appears as an annular plateau over which point sources stand out. These plots also suggest that there is a higher level of emission "inside" the ring (i.e. low "X-dim" values) than "outside" (i.e. high "X-dim" values) and that a rather sharp drop confines the outer limb of the ring. With the limited number of counts detected "inside" the ring, detailed studies of this component are not possible and will have to wait for more sensitive observations.

\subsubsection{Spectrum}

The spectrum of the brightest source in the Cartwheel ring has already been described by Gao et al. (2003). However, with our analysis we find slightly different results; unfortunately not enough details are given in the Gao et al. paper for us to ascertain if the differences are based on different hypotheses or different treatment of the data. Therefore we present here in detail the spectral analysis of the brightest source (the only one for which a spectral analysis can be attempted, with $\sim 380$ net counts) and for the sum of the other individual sources detected around the ring (for which we collect in total more than 600 net counts). The background has been computed in large circles devoid of bright sources at about the same off-axis position as the Cartwheel.

For the brightest source (N.10) we bin the data to have at least 30 total counts in each bin, and we apply a simple powerlaw model with low energy absorption (see Table 2). The fitted $N_{\mathrm{H}}$ value is consistent with other absorption measures in the Carthweel: the best fit is higher than the line-of-sight Galactic value but consistent with the intrinsic absorption measured e.g. in the optical band: the value of $A_{\mathrm{V}}=2$ measured (Fosbury \& Hawarden 1977) corresponds to $N_{\mathrm{H}}=3.8 \times 10^{21} \mathrm{~cm}^{-2}$ (using $A_{\mathrm{V}}=N_{\mathrm{H}} \times 5.3 \times 10^{-22}$, for $R_{\mathrm{V}}=3.1$, see e.g. Bohlin et al. 1978) well within the range of the fitted $N_{\mathrm{H}}$ values in Table 2. The power law slope would indicate a High Mass X-ray Binary (HMXB), as also proposed by Wolter et al. (1999) based on luminosity arguments.

Although a more complex model is not required by the data, we also used the absorbed Multicolor Disk model (diskbb model in XSPEC; Mitsuda et al. 1984) used for other ULX (see e.g. Bauer et al. 2003; Zezas et al. 2002), which gives an equally good fit (see Table 2). The formal errors in the 

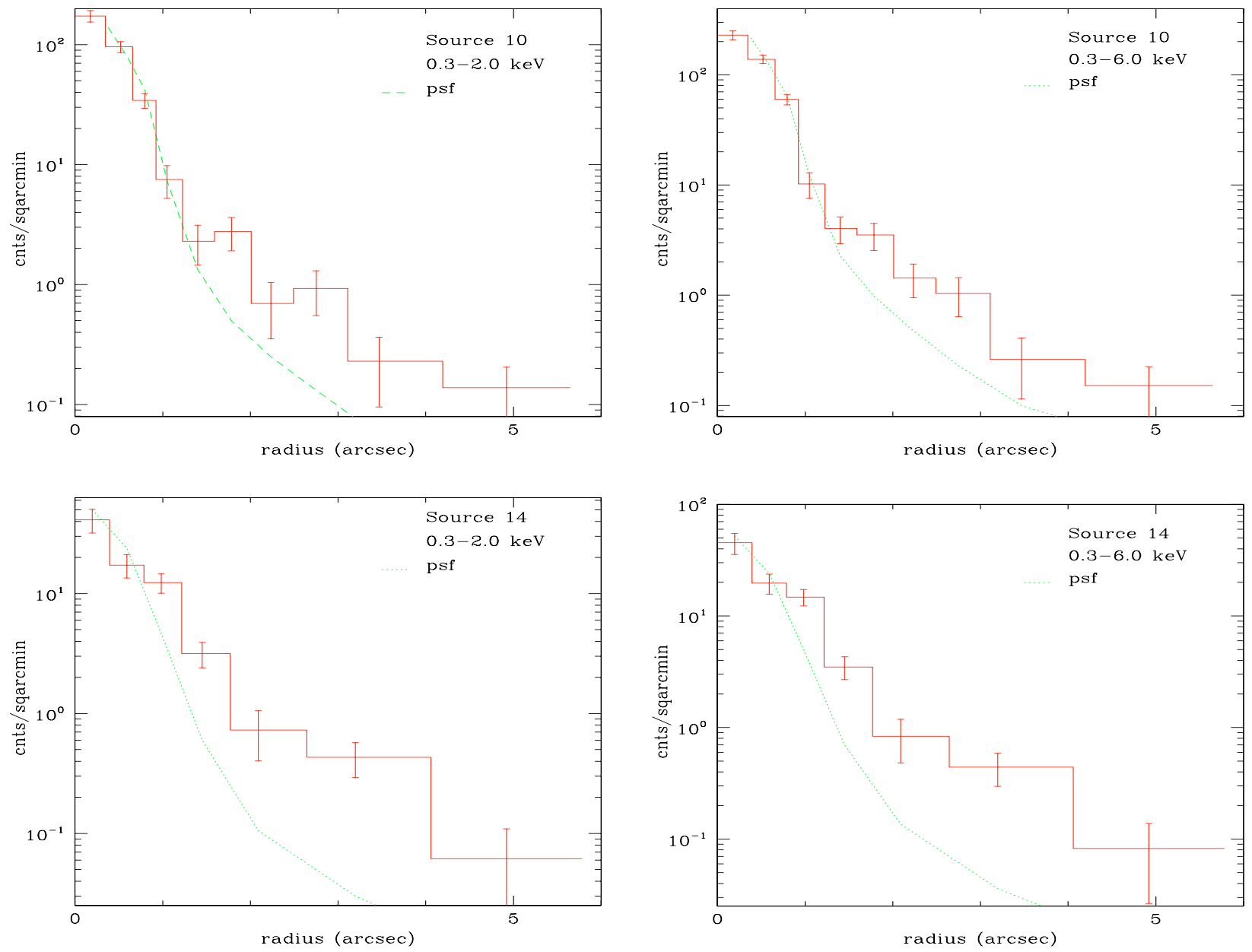

Fig. 3. Surface brightness profile of source N.10 in the soft range $(0.3-2 \mathrm{keV}$ ) (top left) and in the total range (0.3-6 keV) (top right) compared with the PSF from chart in the same energy ranges. Surface brightness profile of source N.14 in the energy range 0.3-2 keV (bottom left) and 0.3-6 keV (bottom right) compared with the PSF from chart in the same energy ranges. Source N.13 has been masked out before computing the profiles.
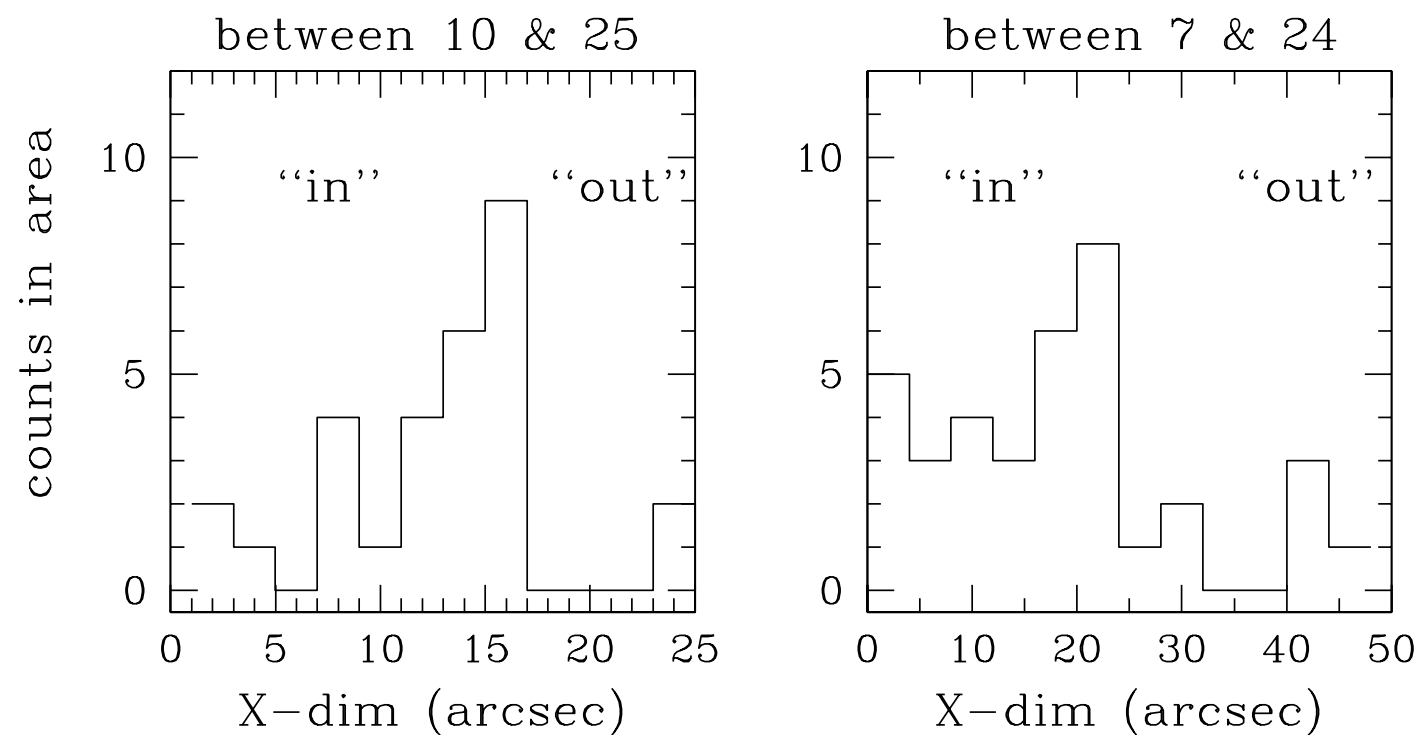

Fig. 4. Cuts in regions orthogonal to the star-forming ring. See text for comments. The regions used are slits of 5.6" and 5.9" (left and right panels respectively) and length given by the $x$-axis. Left: in the gap between the brightest source N.10, and source N.25, i.e. approximately in the South direction; Right: in the gap between sources N.7 and N.24, i.e. approximately oriented NW-SE. 


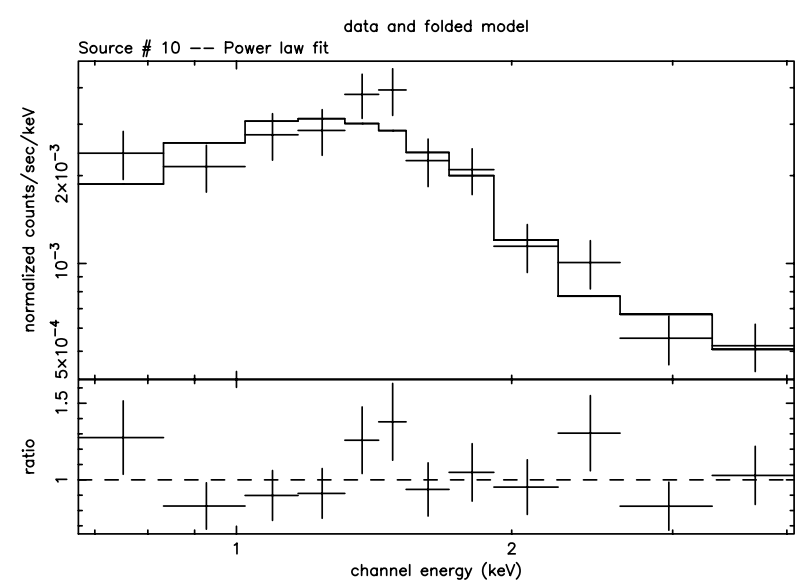

Fig. 5. ACIS-S spectrum of source N.10. The solid line corresponds with a power law model with $\Gamma=1.6$ and low energy absorption $N_{\mathrm{H}}=$ $3.6 \times 10^{21} \mathrm{~cm}^{-2}$ (wabs + pow). The lower panel shows the ratio between data and model.

temperature $/ N_{\mathrm{H}}$ parameters ( $90 \%$ confidence region for one interesting parameter) are given in Table 2.

Unfortunately, given the statistics, a thermal plasma model, like a Raymond-Smith or a Mekal with fixed solar abundance, also gives formally acceptable fits $\left(\chi_{v}^{2}\right.$ of the order of 1.1), with a temperature $(\gtrsim 2.5 \mathrm{keV})$ not strongly constrained. Therefore we feel that more detailed spectral models will not convey meaningful additional information for this source. However, we notice the same small excess at $\sim 1.5 \mathrm{keV}$ that was pointed out by Gao et al. (2003). This line could correspond to a feature from either $\mathrm{Mg}$, or $\mathrm{Al}$, but if these are due to a low temperature plasma, there should be more prominent lines around $1 \mathrm{keV}$. Lines produced by low ionization states of $\mathrm{Mg}, \mathrm{Si}$, and $\mathrm{S}$ in an optically thin gas (see e.g. Iwasawa et al. 2003) could also be an explanation: e.g. in the spectrum of source \#11 in the Antennae a MgXIII line is fitted at $1.50 \mathrm{keV}$ (Zezas et al. 2002). While adding a Gaussian component would clearly reduce the minimum $\chi^{2}$, we feel that this is not required, either statistically (the $\chi_{v}^{2}$ is about 1 even without the line) or from the distribution of the residuals. Moreover it is also clear from the Gao et al. results that their $\chi_{v}^{2}$ are small ( $<1$ always), reflecting the very low significance of each bin.

Fluxes and luminosities are given in Table 2 for different bands, including a luminosity in the $0.05-100 \mathrm{keV}$ range, often assumed to be a measure of the bolometric luminosity. All luminosities are k-corrected. As the values in Table 2 show, the "bolometric" luminosity strongly depends on the model and on the $N_{\mathrm{H}}$ value assumed, given the large extrapolation to both low and high energies, therefore caution should be used when comparing "bolometric" luminosities derived from data extracted in significantly smaller energy ranges and extrapolated using different models.

To derive an average spectrum for the individual sources in the Cartwheel we also accumulated the counts from $\sim 1^{\prime \prime}$ circles around the positions associated to the other individual sources detected. Source N.10 is not included since it is by far the brightest and its inclusion would heavily bias the results.

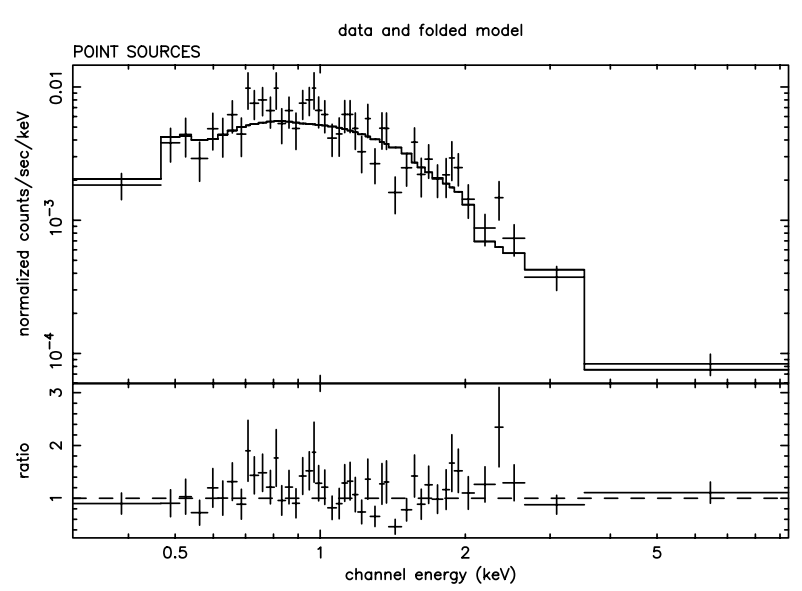

Fig. 6. ACIS-S spectrum of the combined individual sources. The solid line corresponds with a power law fit with $\Gamma=2.2$ and $N_{\mathrm{H}}=$ $1.9 \times 10^{21} \mathrm{~cm}^{-2}$. Source N.10 is not included since it would strongly bias the statistics. Sources N.11 and N.22 are also not considered since they have a very different spectral distribution possibly indicating that they are either background sources or intrinsically absorbed ones.

We also do not include sources N.11 and N.22 that appear to have a lower soft/hard count ratio than the average source, suggesting either an intrinsically different spectrum or a larger absorbing column. Since their location is also not coincident with the star-forming ring, they could be background sources seen through the absorbing material in the Cartwheel. Optical observations (imaging and spectral) are needed to confirm the identification.

The spectral data are binned so that each bin has a significance $\geq 2 \sigma$ after background subtraction. The resulting spectrum is shown in Fig. 6 . The data are fitted by a power law with best fit values $N_{\mathrm{H}}=1.9[1.5-2.3] \times 10^{21} \mathrm{~cm}^{-2}$ and $\Gamma=2.2$ [2.04-2.34] (reduced $\chi^{2}=1.12$ for 50 d.o.f.). Even in this case, the derived low energy absorption is higher than galactic, but consistent with the reddening observed in the HII regions. The contour plot of the uncertainties in spectral index and low energy absorption is shown in Fig. 7. The photon index we obtain is steeper than what is observed in other bright binaries in nearby galaxies when fitted with a simple power law model, and in particular steeper than the spectrum of source N.10, but a few examples of steep sources are present e.g. in the Antennae (Zezas et al. 2002) or in other galaxies (e.g. Humphrey et al. 2003). Since it is likely that this slope results from a combination of different slopes, and perhaps even of different intrinsic absorption, depending on the location of the source with respect to the star forming region, this result should be taken with caution and might not be indicative of a population of steeper spectra sources.

The measured unabsorbed flux is 3.0/2.6 $\times$ $10^{-14} \mathrm{erg} \mathrm{cm}^{-2} \mathrm{~s}^{-1}$ (in the $0.5-2.0 / 2.0-10 \mathrm{keV}$ band), which corresponds to a total luminosity of $5.3 \times 10^{40} / 4.6 \times 10^{40}$ $(0.5-2.0 / 2.0-10.0 \mathrm{keV}) \mathrm{erg} \mathrm{s}^{-1}$. The sum of the luminosity of the detected point sources is then roughly twice that of N.10 in the soft band and comparable to that of N.10 in the hard band. 


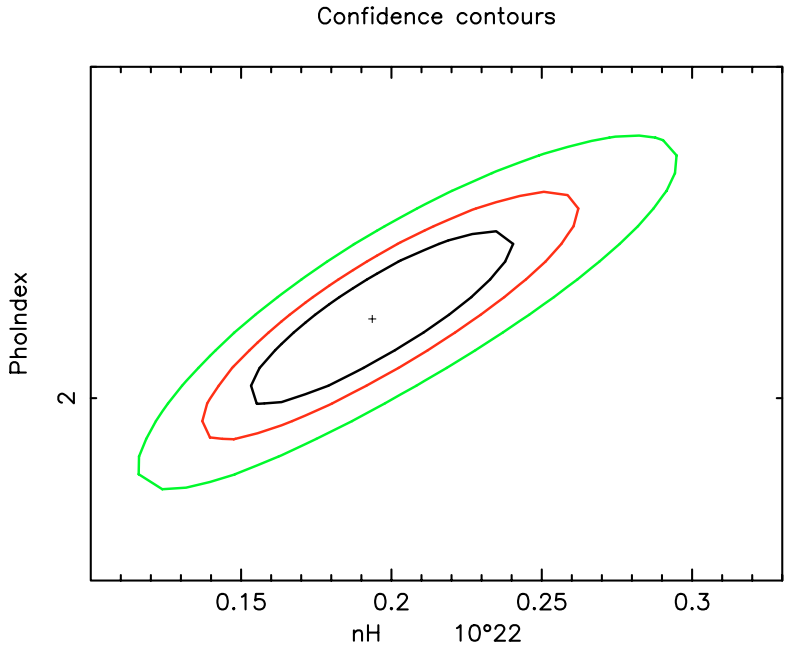

Fig. 7. Confidence contours of the two parameters $\Gamma$ and $N_{\mathrm{H}}$ for the combined point-source spectrum. Curves are at the $68 \%, 90 \%$ and $99 \%$ confidence level for the two parameters.

\subsection{Diffuse component}

As mentioned above, we have also detected a more extended component that coincides with the ring. There is a slight indication of diffuse emission also in the center of the galaxy, but with no direct relation to the other optical structures like the inner ring or the spokes. The smoothed image in the soft band (see Fig. 1) suggests also a low surface brightness emission extending towards galaxies G1 and G2, however, the statistical significance is low. Support for the presence of a diffuse component comes from the plots in Fig. 4, where the average emission inside the ring is higher than outside it. Figure 3 also shows a shoulder at the ring position more pronounced in the softer than in the harder band. We conclude therefore that there might be a hot gaseous component that permeates the Cartwheel and might be enhanced, or more heated, in the ring.

To investigate the spectral properties of the "extended" emission we collect counts from a region that includes virtually all of the Cartwheel extent, but excludes all detected point sources analyzed previously. The spectrum has been binned to have a significance $\geq 2 \sigma$ after background subtraction in each bin. In spite of the limited statistics, we found that the spectrum requires at least two components. A single component fit (e.g. power law, plasma model with fixed abundance) is formally acceptable since the errorbars are large, but it has badly distributed residuals. We tried different combinations of two components, which are all equally acceptable, given the statistics. A good representation (Fig. 8) is given by a combination of a power law $\left(\Gamma=2.3, N_{\mathrm{H}}=2.3 \times 10^{21} \mathrm{~cm}^{-2}\right)$ and a plasma model (Raymond-Smith component with $k T=0.2 \mathrm{keV}$ and an abundance fixed at $0.5 \times$ solar to reflect the low metallicity of the gas measured in optical-IR). The power law component, which could represent fainter unresolved individual binaries, has a slope consistent with that found for the combined point sources (see Sect. 4.1.3). The unabsorbed flux of this component is $F_{\mathrm{x}}=1.1 \times 10^{-14} / 8.5 \times 10^{-15} \mathrm{erg} \mathrm{cm}^{-2} \mathrm{~s}^{-1}$ $(0.5-2.0 / 2.0-10.0 \mathrm{keV})$, about $25 / 10 \%$ of the resolved point source flux in the soft and hard band respectively. The flux of

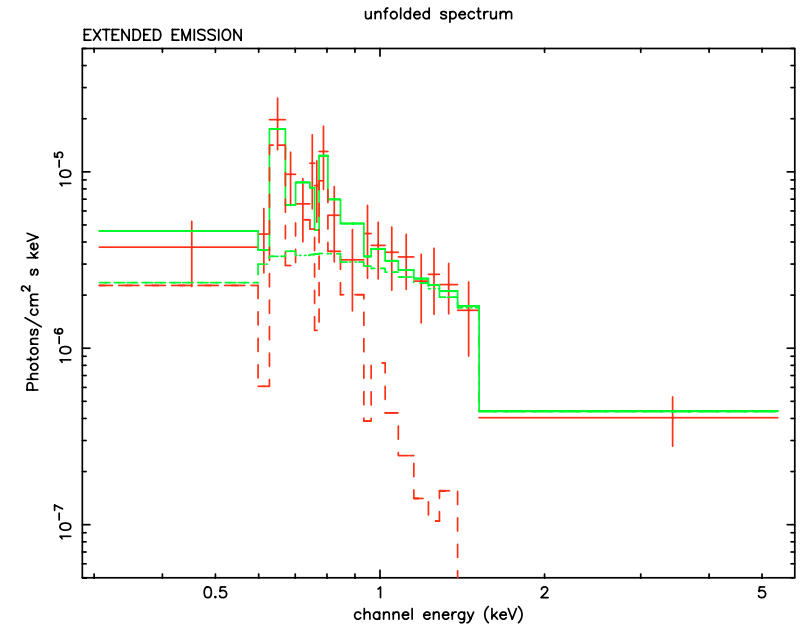

Fig. 8. ACIS-S unfolded spectrum of the diffuse component: even if the statistics are low, the spectrum cannot be fitted by a single component. We show here the fit with a hot plasma component (dashed line) plus power law (dashed gray line) plus the total fit (solid gray line). See text for details.

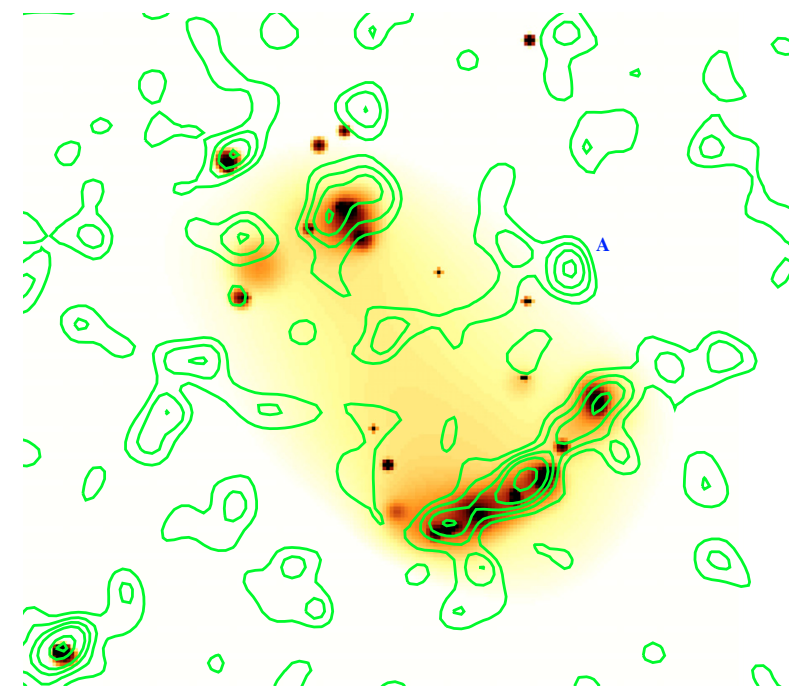

Fig. 9. The ROSAT-HRI contours from the adaptively smoothed image are over-plotted onto the Chandra soft adaptively smoothed image in gray scale.

the diffuse hot gas component is $F_{\mathrm{x}}=1.6 \times 10^{-14} \mathrm{erg} \mathrm{cm}^{-2} \mathrm{~s}^{-1}$ $(0.5-2.0 \mathrm{keV}$ band). It contributes mostly at $0.6-0.9 \mathrm{keV}$ as expected from the temperature found. The total luminosity of the gas is of the order of $\sim 3 \times 10^{40} \mathrm{erg} \mathrm{s}^{-1}$, i.e. only about a factor 4 less than the soft gaseous component in the most X-ray luminous starburst galaxy known, NGC 3256 (Moran et al. 1999).

\section{Discussion}

\subsection{Comparison with literature results}

In Fig. 9 we show the HRI contours overlaid on the adaptively smoothed Chandra image in the soft band. The only striking difference in the image is in source labeled " $A$ " in the figure, which is not present in the new Chandra image. However, the source is probably not related to the Cartwheel itself, 
so it might be any kind of variable source from an AGN to a transient Galactic source. The total soft luminosity seen by Chandra (adding up all the different components, from point sources to diffuse gas) of $L_{\mathrm{X}}(0.2-2.0) \sim 2 \times 10^{41} \mathrm{erg} \mathrm{s}^{-1}$ is consistent with that of the HRI of $2.5 \times 10^{41} \mathrm{cgs}$, once the same $H_{0}$ is assumed, and taking into account the different spectral parameters used to derive the luminosities.

A strict comparison of the contribution from each individual source is not easy, given the very different size of the PSF of the two instruments. However, by comparing the main knots of emission in the HRI with the corresponding regions in Chandra, we notice a variation in the flux level in at least one region. If we make the hypothesis that the region around G1 has not varied, then the region around sources N.10-13-14 shows a factor of $\sim 3$ increase. This is consistent with variability observed in other ULX, if one source only is responsible for the flux increase. Since N.10 is the dominant source we can probably attribute the variation to this one source; otherwise, as already suggested in Sect. 3.1, we should assume that there is a large compact cluster of sources that vary together in a region of $\sim 1.5-2 \mathrm{kpc}$. For comparison, in a similar region in the Antennae, closer and therfore more resolved, there are up to 7 sources brighter than $10^{38} \mathrm{erg} \mathrm{s}^{-1}$, of which only 2 are above $10^{39} \mathrm{erg} \mathrm{s}^{-1}$ (see Fig. 1 and Table 1 of Zezas et al. 2002), which would contribute only about $1 / 10$ of the observed luminosity of source N.10.

The flux variation implies that an origin of the X-ray emission from SNR is probably less likely than the association with an accreting compact object.

Even if N.10 is by far the brightest source in the ring, it is clear from Fig. 9 that the HRI was also detecting the entire ring emission. We estimate in fact that in the $0.2-2.0 \mathrm{keV}$ band, the brightest source N.10 contributes only $\sim 1 / 4$ of the total luminosity. Gao et al. (2003) instead propose that most of the emission detected by the ROSAT HRI is from this source only; however they use a much broader band than the $0.2-2.0 \mathrm{keV}$ that matches the ROSAT HRI energy band.

Giant HII regions and complex structures, typically coincident with peaks of $\mathrm{H} \alpha$ emission, have been observed in actively star-forming objects like the interacting system "The Antennae" (NGC 4038/9; e.g. Fabbiano et al. 2001) as extremely bright $\mathrm{X}$-ray sources, with intrinsic luminosities reaching several $\times 10^{40} \mathrm{erg} \mathrm{s}^{-1}$. To check this association in the Cartwheel, we have plotted the positions of the HII knots as measured by Higdon (1995) on the X-ray image in Fig. 10. The positions of the circles that mark the HII regions have been shifted by about $1^{\prime \prime}$ in RA and $0.5^{\prime \prime}$ in Dec for better agreement with the locus of the X-ray peaks (well within the positional uncertainty of both the X-ray and the HII reference frame). A general trend in the location of the X-ray and HII emission is evident. However, there is no one-to-one correspondence with any of the X-ray sources. If anything, the X-ray emission seems to be at the edge of the HII knots. The same kind of general positional agreement is evident between the X-ray emission and the Mid-IR peaks (Charmandaris et al. 1999); the so called "hot spot", especially at $15 \mu \mathrm{m}$, dominates the output in this energy range. It coincides with two large HII complexes, but not with the brightest X-ray source. The resolution of the

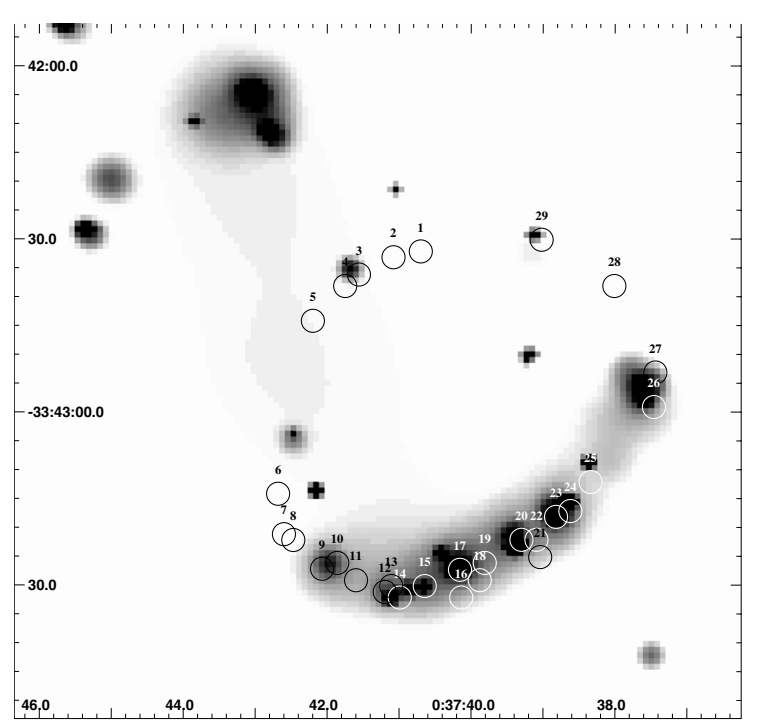

Fig. 10. X-ray smoothed map in the (0.3-7.0) keV band. Over-plotted are the position of HII regions numbered as in Higdon (1995).

ISO data is however 6-7", so more than one X-ray source (e.g. at least N.22 and N.24) might be associated with the ISO emission. The best interpretation of this similarity is that the region is active in general, but time scales and regions of emission are not directly linked.

\subsection{Individual sources}

The excessive number of very high X-ray luminosity individual sources makes it interesting and complicated to understand their nature.

The limiting luminosity for point sources is of the order of $5 \times 10^{38} \mathrm{erg} \mathrm{s}^{-1}$, which is already above the Eddington limit for a neutron star binary $\left(L_{\mathrm{X}} \sim 3 \times 10^{38} \mathrm{erg} \mathrm{s}^{-1}\right.$; see e.g. King et al. 2001). Most of the detected sources are also above the limits of canonical Ultra-Luminous X-ray sources (ULX), i.e. $L_{\mathrm{X}} \geq 10^{39} \mathrm{erg} \mathrm{s}^{-1}$. The main uncertainty in the $L_{\mathrm{X}}$ calculation is the correct association of a few of the individual sources to the Cartwheel itself (i.e. they might be foreground or background sources). We assume that all sources within the optical ring belong to the Cartwheel. However there are also a number of sources not positionally coincident with the ring: N.6, N.8, N.11, N.20, N.22 that we consider below one by one.

N.8 is located between the Cartwheel and the G1 galaxy, so it is probably unrelated to either source, unless the encounter affected even this area. We consider this unlikely and treat it as a background source.

From comparison of the soft and hard count rates sources N.11 and N.22 appear to have a different spectrum from the rest. If this results from higher absorption, with the limited statistics available, we estimate an absorber of $\leq 10^{22} \mathrm{~cm}^{-2}$, consistent with a galaxy like the Cartwheel itself. However it is not possible to determine whether the sources are embedded in the absorber, and therefore belong to the Cartwheel, or are behind it, and therefore background sources. Given the location of N.22 in the ring we consider this to belong to the Cartwheel. The association of N.11 is less certain, however by 


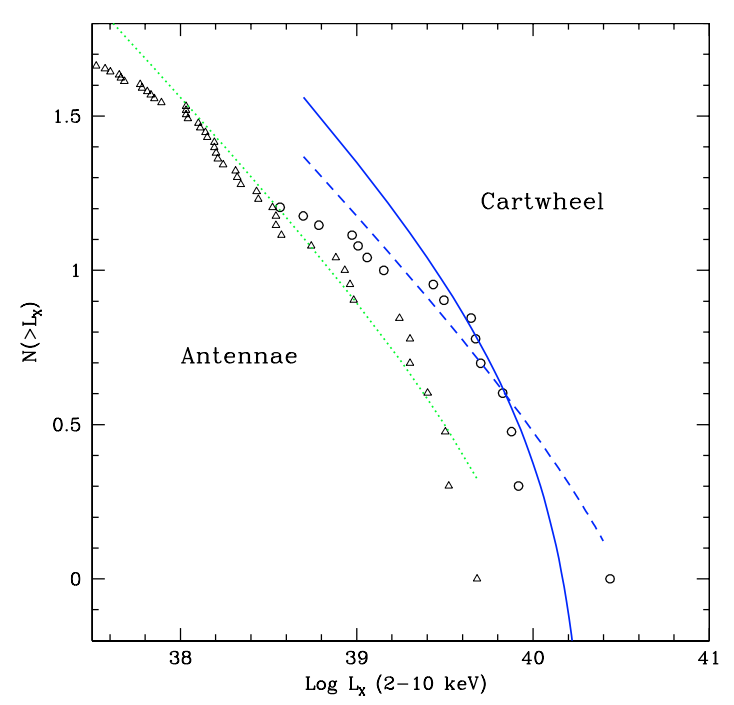

Fig. 11. Luminosity function of all bright isolated sources in the ring. The solid line represents the Grimm et al. (2003) luminosity function with $S F R=20 M_{\odot} \mathrm{yr}^{-1}$ and cut-off luminosity of $L_{\mathrm{X}}=2 \times$ $10^{40} \mathrm{erg} \mathrm{s}^{-1}$. The dashed line has $S F R=12 M_{\odot} \mathrm{yr}^{-1}$ and cut-off luminosity of $L_{\mathrm{X}}=1 \times 10^{41} \mathrm{erg} \mathrm{s}^{-1}$. The Antennae LF from Zezas et al. (2002) is also plotted for comparison, with the original fit from Grimm et al. (2003). We have assumed a constant factor of 0.5 to convert luminosities from the original $0.1-10 \mathrm{keV}$ band to the $2-10 \mathrm{keV}$ band considered here.

analogy with N.22 we also consider it as part of the galaxy and include both in the estimate of the Luminosity Function in the next section.

N.6 and N.20 are not exactly on the peak of star formation, but close to the inner side of the ring. We consider them "leftovers" from past star formation, and therefore related to the Cartwheel.

We discuss below the properties of the ULX in the Cartwheel on a statistical basis, by deriving their Luminosity Function.

\subsection{Luminosity function of ULX in the Cartwheel ring}

We have computed the luminosity function (LF) of all 16 isolated sources detected along the outer ring, assuming the distance of the Cartwheel for computing the luminosities (sources that are not used are indicated with a note in Table 1).

Grimm et al. (2003) propose that a "universal" LF for HMXB can be constructed by normalizing to the Star Formation Rate (SFR) in the formula

$N(>L)=5.4 \operatorname{SFR}\left(L_{38}^{-0.61}-2 \times 10^{-0.61}\right)$

(see their Eq. (7)). The resulting differential LF has a slope of $\alpha=1.61$ and a cut-off luminosity of $L_{\mathrm{X}}(2-10 \mathrm{keV})=$ $2.1 \times 10^{40} \mathrm{erg} \mathrm{s}^{-1}$. We plot the histogram of the LF in Fig. 11 and compare it with the expectation from the Grimm et al. (2003) formula with a value of $S F R=20 M_{\odot} \mathrm{yr}^{-1}$, and with the Antennae LF (from Zezas et al. 2002) with the original fit of the Grimm et al. (2003) formula.

We notice: 1) The slope 1.61 reproduces reasonably well the distribution, above $L_{\mathrm{x}} \sim 10^{39} \mathrm{erg} \mathrm{s}^{-1} ; 2$ ) the low luminosity flattening might be due to incompleteness (lower flux sources are harder and harder to detect above a diffuse emission plateau, see e.g. discussion in Kim \& Fabbiano 2003); 3 ) the cut-off luminosity should be higher than in the Grimm et al. formula: at least source N.10 is above the assumed cut-off; 4) with a cut-off at $L_{X} \sim 1 \times 10^{41} \mathrm{erg} \mathrm{s}^{-1}$, if nothing else should change in the functional form of the above equation, the nominal $S F R$ derived is $\sim 12 M_{\odot} \mathrm{yr}^{-1}$; 5) discarding source N.10 a resonable fit is obtained with the Grimm et al. formula with an $S F R=\sim 20 M_{\odot} \mathrm{yr}^{-1}$; the discrepancy at low luminosities however is larger.

Low Mass X-ray Binaries (LMXB) are unlikely to contribute significantly at these luminosities, since their average LF (Gilfanov 2004) is very steep above a few $10^{37} \mathrm{erg} \mathrm{s}^{-1}$ and has a cut-off at $L_{\mathrm{x}} \sim 2 \times 10^{39} \mathrm{erg} \mathrm{s}^{-1}$, so that their contribution in the luminosity range probed by this observation would be marginal relative to HMXB.

By following the different approach suggested by Grimm et al. (2003) of relating the SFR to the total X-ray luminosity (their Eq. (21)) we derived a slightly higher value of $\sim 25 M_{\odot} \mathrm{yr}^{-1}$. These results compare to the values derived earlier, based on the efficiency of star forming galaxies of producing X-rays (Wolter et al. 1999) of $\dot{M} \sim 20-50 M_{\odot} \mathrm{yr}^{-1}$.

The higher SFR might explain the difference in luminosity of the sources found in the Cartwheel with respect e.g. to those in the Antennae, that have an SFR of 7.1 $M_{\odot} \mathrm{yr}^{-1}$ (Zezas et al. 2002; Fig. 11). The question of a higher cut-off $L_{X}$ in this system remains open.

\section{Summary and conclusion}

We have presented results from a Chandra observation of the Cartwheel. A number of isolated and very luminous sources is present, closely related to the region of high star formation detected at other wavelengths, accounting for at least $75-80 \%$ of the total luminosity of $L_{X}=2.2 \times 10^{41} \mathrm{erg} \mathrm{s}^{-1}$ in the $0.5-10 \mathrm{keV}$ band.

A more extended gaseous component coincident with the ring is also detected, and also a more tentative diffuse component that might permeate the entire system. The extended component has a low temperature $(k T \sim 0.2 \mathrm{keV})$ consistent with an origin related to starburst superwinds as in NGC 3256 (Moran et al. 1999; Lira et al. 2002) or in NGC 253 (Pietsch et al. 2001; Strickland et al. 2000) and a luminosity $L_{X} \sim$ $3 \times 10^{40} \mathrm{erg} \mathrm{s}^{-1}$ in the $0.5-10 \mathrm{keV}$ band.

Individual sources are consistent with being pointlike, although even with the superb Chandra resolution we only probe the kpc scale at the Cartwheel distance. However several considerations prompt us to suggest that we are really detecting individual very bright sources, among the brightest ULX seen in external galaxies. The most luminous, N.10, has a $(0.5-2 / 2-10 \mathrm{keV}) L_{\mathrm{X}}>2 . / 7 . \times 10^{40} \mathrm{erg} \mathrm{s}^{-1}$, to be compared with e.g. the brightest ULX in M $82\left(L_{X} \sim 9 \times 10^{40} \mathrm{erg} \mathrm{s}^{-1}\right.$ in $0.5-10 \mathrm{keV}$ at its brightest; Matsumoto et al. 2001 and Kaaret et al. 2001), in NGC $4559\left(L_{\mathrm{X}}=2 . \times 10^{40} \mathrm{erg} \mathrm{s}^{-1}\right.$ in $0.3-10 \mathrm{keV}$ band; Soria et al. 2004) or in NGC $2276\left(L_{X}=3 . \times 10^{40} \mathrm{erg} \mathrm{s}^{-1}\right.$ in the 0.5-2 keV band; Davis \& Mushotzky 2004). All these luminosities are computed assuming isotropic emission; the true luminosity might be lower if the X-rays are beamed. The crude 
spectral analysis of the brightest source indicates that models typical of accreting binaries (like an absorbed power law or a multicolor disk model) could describe the data. Further, a possible flux variation strongly suggests that the observed emission is due to a single object.

The total luminosity of the individual sources reaches at least $L_{\mathrm{X}}=1.8 \times 10^{41} \mathrm{erg} \mathrm{s}^{-1}$ in the $0.5-10.0 \mathrm{keV}$ band. The derived Luminosity Function is consistent with a population of HMXB in an actively star forming object and the system requires an SFR of at least $12-20 M_{\odot} \mathrm{yr}^{-1}$, in agreement with previous estimates for the Cartwheel. We find a good match with the assumption of the "universal" Luminosity Function for HMXB of Grimm et al. (2003); however we suggest a cut-off luminosity $\sim 5 \times$ higher.

The nature of ULX is still not clear and the wide range of their characteristics points to the possibility that they are a heterogeneous class, as indicated by close scrutiny of nearby objects (e.g., Roberts et al. 2004). We are inclined to exclude SNR because of possible long term variability. Also, the spectral properties are consistent with HMXB. Since the luminosity is so high, a black hole accreting object is more likely than a neutron star. However, no fit with MD gives a low enough temperature (the best fit is $k T=1.3 \mathrm{keV}$ ) to indicate a very high mass compact object.

Our forthcoming XMM-Newton observation will allow us to confirm the presence of the diffuse component with higher statistical significance and to better define the nature of the individual sources through spectral analysis.

Acknowledgements. We thank the referee for comments that greatly improved the presentation of this work. We have received partial financial support from the Italian Space Agency (ASI). This research has made use of the NASA/IPAC Extragalactic Database (NED) which is operated by the Jet Propulsion Laboratory, California Institute of Technology, under contract with the National Aeronautics and Space Administration. This research has made use of SAOImage DS9, developed by Smithsonian Astrophysical Observatory.

\section{References}

Appleton, P. N., \& Struck-Marcell, C. 1987, ApJ, 318, 103 Athanassoula, E., Puerari, I., \& Bosma, A. 1997, MNRAS, 286, 284 Bauer, F. E., Brandt, W. N., \& Lehmer, B. 2003, AJ, 126, 2797 Bohlin, R. C., Savage, B. D., \& Drake, J. F. 1978, ApJ, 224, 132
Charmandaris, V., Laurent, O., Mirabel, I. F., et al. 1999, A\&A, 341, 69

Davis, S. D., \& Mushotzky, R. F. 2004, ApJ, 604, 653

Fabbiano, G., Zezas, A., \& Murray, S. S. 2001, ApJ, 554, 1035

Fosbury, R. A., \& Hawarden, T. G. 1977, MNRAS, 178, 473

Gao, Y., Wang, Q. D., Appleton, P. N., \& Lucas, R. A. 2003, APJ, 596, 171

Gilfanov, M. 2004, MNRAS, 349, 146

Grimm, H.-J., Gilfanov, M., \& Sunyaev, R. 2003, MNRAS, 339, 793

Higdon, J. L. 1995, ApJ, 455, 524

Higdon, J. L. 1996, ApJ, 467, 241

Humphrey, P. J., Fabbiano, G., Elvis, M., Church, M. J., \& Balucinska-Church 2003, MNRAS, 344, 134

Iovino, A., de Carvalho, R. R., Gal, R. R., et al. 2003, AJ, 125, 1660

Iwasawa, K., Wilson, A. S., Fabian, A. C., \& Young, A. J. 2003, MNRAS, 345, 369

Kaaret, P., Prestwich, A. H., Zezas, A., et al. 2001, MNRAS, 321, L29

Kim, D.-W., \& Fabbiano, G. 2003, ApJ, 586, 826

King, A. R., Davies, M. B., Ward, M. J., Fabbiano, G., \& Elvis, M. 2001, ApJ, 552, L109

Lira, P., Ward, M., Zezas, A., Alonso-Herrero, A., \& Ueno, S. 2002, MNRAS, 330, 259

Marcum, P. M., Appleton, P. N., \& Higdon, J. 1992, ApJ, 399, 57

Markevitch, M. 1998, ApJ, 504, 27

Matsumoto, H., Tsuru, T. G., Koyama, K., et al. 2001, ApJ, 547, 25

Mitsuda, K., Inoue, H., Koyama, K., et al. 1984, PASJ, 36, 741

Moran, E. C., Lehnert, M. D., \& Helfand, D. J. 1999, ApJ, 526, 649

Pietsch, W., Roberts, T. P., Sako, M., et al. 2001, A\&AL, 365, 174

Roberts, T. P., Warwick, R. S., Ward, M. J., \& Goad, M. R. 2004, MNRAS, 350, 1536

Rosati, P., Tozzi, P., Giacconi, R., et al. 2002, ApJ, 566, 667

Soria, R., Cropper, M., \& Pakull, M. 2003, Compact Binaries in the Galaxy and Beyond, La Paz, Mexico, IAU Coll., 194 [arXiv: astro-ph/0312539]

Strickland, D. K., Heckman, T. M., Weaver, K. A., \& Dahlem, M. 2000, AJ, 120, 2965

Struck, C., Appleton, P. N., Borne, K. D., \& Lucas, R. A. 1996, AJ, 112,1868

Taylor, K., \& Atherton, P. D. 1984, MNRAS, 208, 601

Theys, J. C., \& Spieghel, A. E. 1976, ApJ, 208, 650

Weisskopf, M., Tananbaum, H., Van Speybroek, L., \& O’Dell, S. 2000, Proc. SPIE, 4012, 2, ed. J. E. Truemper, \& B. Aschenbach

Wolter, A., Trinchieri, G., \& Iovino, A. 1999, A\&A, 342, 41

Wolter, A., \& Trinchieri, G. 2003, Mem. Soc. Astron. It. Suppl., 3, 273

Zanichelli, A., Vigotti, M., Scaramella, R., Grueff, G., \& Vettolani, G. 2001, A\&A, 379, 21

Zezas, A., Fabbiano, G., Rots, A., \& Murray, S. 2002, ApJS, 142, 239 
A. Wolter and G. Trinchieri: The X-ray Cartwheel, Online Material p 1

\section{Online Material}




\section{Appendix A}

Table A.1 lists all sources detected in the ACIS-S CCD7 field detected by the wavelet algorithm (see Sect. 3.1). The table lists the CXO name, the X-ray position, the net counts and their errors from the wavelet analysis, the significance of detection and the flux in the $0.5-10 \mathrm{keV}$ band for all the sources detected in the entire field of view of the CCD (S3). Fluxes are computed for all sources by assuming Galactic $N_{\mathrm{H}}=2 . \times 10^{20} \mathrm{~cm}^{-2}$ and a power law with index $\Gamma=1.7$. Table A.1 also includes the sources already presented in Table 1 since the spectral assumptions in the two tables are different; notes in the last column indicate the number the sources have in Table 1 and other names from NED.

The wavelet algorithm detects 72 sources in the S3 CCD area $\left(8.4^{\prime \prime} \times 8.4^{\prime \prime}\right), 47$ of which are not positionally related to the Cartwheel group. The density of the sources corresponds therefore to $\sim 2.3 \times 10^{3}$ sources/sq. deg at the faintest detected

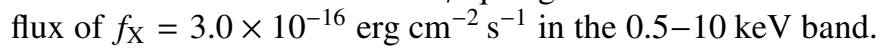
This is entirely consistent with the density measured in the deep Chandra surveys (e.g. Rosati et al. 2002).

None of these field sources is identified in the literature although several optical/radio associations are possible (e.g. PKS or NVSS sources, faint optical counterpart visible on the POSS, or even with magnitude from APM, etc.) We discuss here briefly the X-ray properties of the brighter ones, for which we have explored the spectral properties (i.e. those sources with more than 500 net counts).

We investigate in detail the case of CXO J003728.8334442 , the brightest source detected in the area, which is positionally coincident with the radio source PKS 0035-340, but so far not identified. The statistics of the X-ray data allows us to collect a spectrum, which we bin in such a way that each channel has $\geq 2 \sigma$ significance after background subtraction (Fig. A.1). A single power law model gives an acceptable fit with a photon index $\Gamma=1.72$ [1.63-1.78] and $N_{\mathrm{H}}=6.1[4.2-8.0] \times 10^{20} \mathrm{~cm}^{-2}$, consistent with the classical AGN spectrum, and unabsorbed flux (0.5-10 keV band) $f_{\mathrm{X}}=1.3 \times 10^{-13} \mathrm{erg} \mathrm{cm}^{-2} \mathrm{~s}^{-1}$. As is evident from the figure, even if the $\chi^{2}$ is statistically acceptable, there is an excess at $\sim 4.5 \mathrm{keV}$. If we interpret this feature as a Fe-K $\alpha$ line, we can use it to measure the still unknown redshift of the source. We can make two different hypotheses that imply two different spectral models: that the source is an AGN (the radio galaxy itself); or that the X-ray source is a cluster of galaxies, of which the radio galaxy is a tracer (see e.g., Zanichelli et al. 2001, who however did not find a cluster around PKS0035-340). In either case, the interpretation of the feature as the Fe-K $\alpha$ line gives a similar $z \sim 0.45$. An even better match with the spectral feature is obtained by fitting both the $6.4 \mathrm{keV}$ ("neutral") and the $6.7 \mathrm{keV}$ ("highly-ionized") Fe-line at the same redshift. The significance of each line is only about $1 \sigma$; nevertheless we obtain a consistent fit with $z=0.425$, and equivalent width of $\sim 200 \mathrm{eV}$ for both lines $(<500 \mathrm{eV}$ at the $90 \%$ confidence level). The derived luminosity would then be $L_{\mathrm{X}}=$ $8.5 \times 10^{43} \mathrm{erg} \mathrm{s}^{-1}$ (for $H_{0}=75 \mathrm{~km} \mathrm{~s}^{-1} \mathrm{Mpc}^{-1}$, or $L_{\mathrm{X}}=$ $1.9 \times 10^{44} \mathrm{erg} \mathrm{s}^{-1}$ for $H_{0}=50 \mathrm{~km} \mathrm{~s}^{-1} \mathrm{Mpc}^{-1}$ ), consistent both with the AGN and the cluster hypothesis. The formal

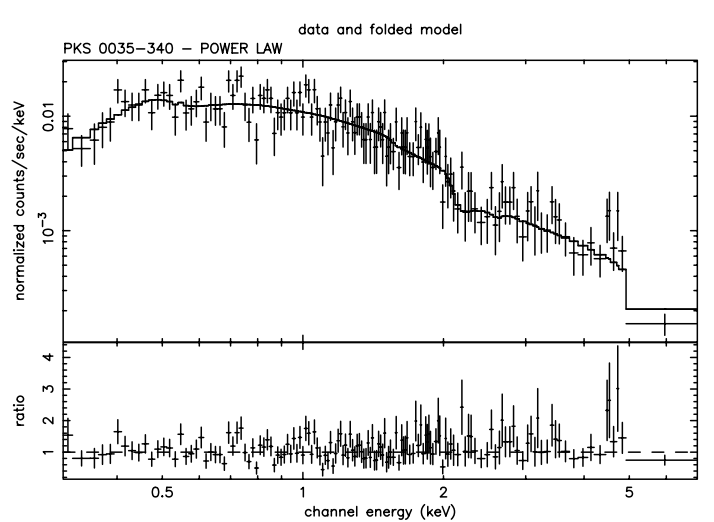

Fig. A.1. PKS0035-340 spectrum fitted with a Power law model. The excess at $\sim 4.5 \mathrm{keV}$ is evident

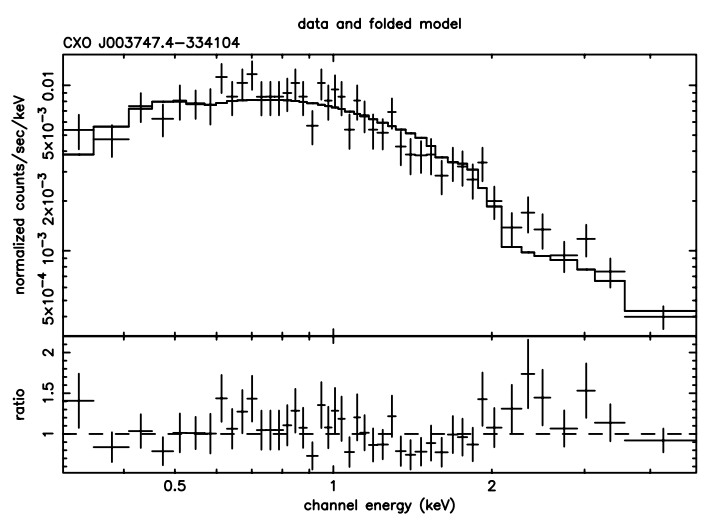

Fig. A.2. The spectrum of CXO J003747.4-334104 fitted with a power law model.

temperature fit of $k T=6.8$ [5.7-8.3] keV is broadly consistent with the expectation from the luminosity - temperature relation of Markevitch (1998).

To our knowledge this is the first redshift derived from $\mathrm{X}$-ray spectroscopy without prior measures or estimates. The statistics is however scanty and does not grant that the interpretation of the feature is correct.

We further analyzed the spectral data of CXO J003747.4334104 and CXO J003756.3-334124.

CXO J003747.4-334104 has a faint optical counterpart visible on the DSS II red plate. A single power law model with Galactic $N_{\mathrm{H}}$ gives an acceptable fit with a photon index $\Gamma=$ 1.75 [1.66-1.85], consistent with the canonical AGN spectrum, and flux $(0.5-10 \mathrm{keV}) f_{\mathrm{X}}=8.8 \times 10^{-14} \mathrm{erg} \mathrm{cm}^{-2} \mathrm{~s}^{-1}$. If the source is indeed an AGN with redshift in the range 0.4-2.0 (typical of X-ray selected AGN) the derived luminosity would then be $L_{X}(0.5-10 \mathrm{keV})=0.5-20 \times 10^{44} \mathrm{erg} \mathrm{s}^{-1}$ (for $\left.H_{0}=75 \mathrm{~km} \mathrm{~s}^{-1} \mathrm{Mpc}^{-1}\right)$, consistent with the AGN hypothesis. If the small excess at $\sim 2.4 \mathrm{keV}$ were the $\mathrm{Fe} \mathrm{K} \alpha$ line, than the redshift would be $z \sim 1.7$.

CXO J003756.3-334124 does not have an optical counterpart on the DSS II plate, and therefore the most likely counterpart is a distant X-ray cluster. The spectrum would then need to be fitted with a thermal or plasma emission model; however, not knowing the redshift this could be tricky. If we use a bremsstrahlung model the best fit temperature is 
A. Wolter and G. Trinchieri: The X-ray Cartwheel, Online Material p 3

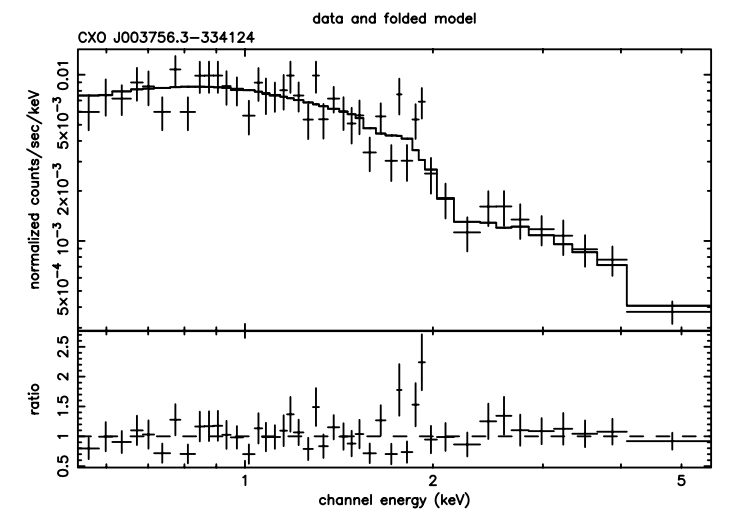

Fig. A.3. The spectrum of CXO J003756.3-334124 fitted with a bremsstrahlung model.

$k T=9.4[6.5-15.6] \mathrm{keV}$ with a flux $(0.5-10 \mathrm{keV}) f_{\mathrm{X}}=$

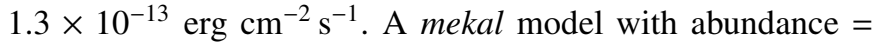
0.3 solar, at a few selected redshifts between 0.5 and 1.2, also gives a reasonable fit and a similar flux. For the same redshift range 0.5 to 1.2 (typical of $\mathrm{X}$-ray selected clusters of galaxies) the derived luminosity would then be $L_{X}(0.5-10 \mathrm{keV})=$ 1. $-8 \times 10^{44} \mathrm{erg} \mathrm{s}^{-1}$ (for $H_{0}=75 \mathrm{~km} \mathrm{~s}^{-1} \mathrm{Mpc}^{-1}$ ), in the range of distant cluster luminosities.

A single power law could also fit the data within the errors, and results in a slope $\Gamma=1.42[1.31-1.53]$, flatter than classical AGN spectra.

For these two sources no conclusion can be drawn from $\mathrm{X}$-rays alone, and a detailed optical investigation would be needed to define the counterpart. 
Table A.1. Sources detected by the wavelet algorithm in the S3 field of view. Count rates have been converted into flux by assuming a power law with index $\Gamma=1.7$ and Galactic low energy absorption $N_{\mathrm{H}}=2 \times 10^{20} \mathrm{~cm}^{-2}$. An X-ray spectral analysis is presented in the text for sources indicated with *.

\begin{tabular}{|c|c|c|c|c|c|c|}
\hline $\begin{array}{l}\text { Name } \\
\text { CXOJ- }\end{array}$ & \multicolumn{2}{|c|}{$\mathrm{J} 2000$} & Net cts & $S / N$ & $\begin{array}{r}\text { Flux }\left(\mathrm{erg} \mathrm{cm}^{-2} \mathrm{~s}^{-1}\right) \\
(0.5-10 \mathrm{keV})\end{array}$ & Other names \\
\hline $003724.1-334309$ & 03724.14 & -334309.02 & $131.24 \pm 11.62$ & 42.03 & $1.3 \times 10^{-14}$ & \\
\hline $003727.1-334245$ & 03727.11 & -334245.61 & $6.17 \pm 2.65$ & 2.73 & $5.9 \times 10^{-16}$ & \\
\hline $003727.6-334347$ & 03727.61 & -334347.78 & $10.97 \pm 3.46$ & 4.70 & $1.0 \times 10^{-15}$ & \\
\hline $003728.2-334407$ & 03728.26 & -334407.18 & $49.61 \pm 7.21$ & 17.89 & $4.7 \times 10^{-15}$ & \\
\hline $003728.8-334442^{*}$ & 03728.83 & -334442.26 & $1500.78 \pm 38.92$ & 308.23 & $1.4 \times 10^{-13}$ & PKS 0035-340 \\
\hline $003729.6-334638$ & 03729.68 & -334638.82 & $6.73 \pm 3.00$ & 2.46 & $6.4 \times 10^{-16}$ & \\
\hline $003731.5-334404$ & 03731.55 & -334404.83 & $15.42 \pm 4.12$ & 6.11 & $1.5 \times 10^{-15}$ & \\
\hline $003733.0-334338$ & 03733.00 & -334338.41 & $21.40 \pm 4.80$ & 8.45 & $2.0 \times 10^{-15}$ & \\
\hline $003733.6-334543$ & 03733.69 & -334543.68 & $12.63 \pm 3.74$ & 5.14 & $1.2 \times 10^{-15}$ & \\
\hline $003734.0-334601$ & 03734.06 & -334601.24 & $12.66 \pm 3.74$ & 5.18 & $1.2 \times 10^{-15}$ & \\
\hline $003735.1-334517$ & 03735.14 & -334517.32 & $247.79 \pm 15.94$ & 68.12 & $2.4 \times 10^{-14}$ & \\
\hline $003737.3-334515$ & 03737.33 & -334515.88 & $27.06 \pm 5.39$ & 10.25 & $2.6 \times 10^{-15}$ & \\
\hline $003737.5-334342$ & 03737.54 & -334342.09 & $8.12 \pm 3.00$ & 3.56 & $7.8 \times 10^{-16}$ & \\
\hline $003737.5-334256$ & 03737.58 & -334256.94 & $116.10 \pm 10.95$ & 36.78 & $1.1 \times 10^{-14}$ & N.17 \\
\hline $003737.6-334254$ & 03737.60 & -334254.95 & $94.51 \pm 9.90$ & 30.90 & $9.0 \times 10^{-15}$ & N.16 \\
\hline $003738.3-334308$ & 03738.32 & -334308.72 & $16.06 \pm 4.24$ & 6.08 & $1.5 \times 10^{-15}$ & N.15 \\
\hline $003738.7-334316$ & 03738.71 & -334316.08 & $105.92 \pm 10.54$ & 31.01 & $1.0 \times 10^{-14}$ & N.14 \\
\hline $003738.7-334318$ & 03738.78 & -334318.66 & $38.02 \pm 6.40$ & 12.96 & $3.6 \times 10^{-15}$ & N.13 \\
\hline $003738.9-334517$ & 03738.98 & -334517.73 & $14.26 \pm 4.00$ & 5.53 & $1.4 \times 10^{-15}$ & \\
\hline $003739.0-334118$ & 03739.06 & $-3341 \quad 18.93$ & $22.50 \pm 4.90$ & 9.00 & $2.1 \times 10^{-15}$ & \\
\hline $003739.1-334229$ & 03739.15 & -334229.57 & $43.75 \pm 6.78$ & 16.02 & $4.2 \times 10^{-15}$ & N.12 \\
\hline $003739.1-334123$ & 03739.16 & -334123.42 & $7.74 \pm 3.00$ & 3.20 & $7.4 \times 10^{-16}$ & \\
\hline $003739.2-334250$ & 03739.21 & -334250.10 & $62.59 \pm 8.06$ & 22.54 & $6.0 \times 10^{-15}$ & N.11 \\
\hline $003739.3-334323$ & 03739.38 & -334323.07 & $383.77 \pm 19.77$ & 100.35 & $3.7 \times 10^{-14}$ & N.10 \\
\hline $003740.1-334326$ & 03740.15 & -334326.01 & $5.16 \pm 2.45$ & 2.28 & $4.9 \times 10^{-16}$ & N.24 \\
\hline $003740.4-334324$ & 03740.44 & -334324.87 & $14.28 \pm 4.00$ & 5.56 & $1.4 \times 10^{-15}$ & N.22, NVSS J003740-334324 \\
\hline $003740.4-334013$ & 03740.45 & -334013.11 & $71.40 \pm 8.60$ & 25.24 & $6.8 \times 10^{-15}$ & \\
\hline $003740.8-334330$ & 03740.86 & -334330.85 & $66.20 \pm 8.31$ & 22.96 & $6.3 \times 10^{-15}$ & N.9 \\
\hline $003741.0-334221$ & 03741.03 & -334221.37 & $21.23 \pm 4.80$ & 8.20 & $2.0 \times 10^{-15}$ & N. 8 \\
\hline $003741.0-334331$ & 03741.08 & -334331.81 & $70.64 \pm 8.60$ & 23.34 & $6.7 \times 10^{-15}$ & N.7 \\
\hline $003741.2-334232$ & 03741.23 & -334232.08 & $6.97 \pm 2.83$ & 2.99 & $6.7 \times 10^{-16}$ & N.21 \\
\hline $003741.9-334326$ & 03741.98 & -334326.36 & $8.54 \pm 3.16$ & 3.43 & $8.2 \times 10^{-16}$ & N.23 \\
\hline $003742.1-334313$ & 03742.11 & -334313.72 & $13.20 \pm 3.87$ & 5.08 & $1.3 \times 10^{-15}$ & N.20 \\
\hline $003742.3-334122$ & 03742.39 & -334122.80 & $7.92 \pm 3.00$ & 3.37 & $7.6 \times 10^{-16}$ & \\
\hline 003742.4-334304 & 03742.47 & -334304.18 & $19.97 \pm 4.69$ & 7.48 & $1.9 \times 10^{-15}$ & N.6 \\
\hline 003742.7-334212 & 03742.79 & -334212.46 & $40.58 \pm 6.63$ & 13.34 & $3.9 \times 10^{-15}$ & N.5, G1 \\
\hline 003742.8-334209 & 03742.84 & -334209.63 & $18.57 \pm 4.58$ & 6.67 & $1.8 \times 10^{-15}$ & N.19, G1 \\
\hline $003743.0-334020$ & 03743.05 & -334020.75 & $21.09 \pm 4.80$ & 8.02 & $2.0 \times 10^{-15}$ & \\
\hline $003743.0-334205$ & 03743.01 & -334205.96 & $48.56 \pm 7.14$ & 17.43 & $4.6 \times 10^{-15}$ & N.4, G1 \\
\hline $003743.1-334142$ & 03743.10 & -334142.91 & $12.45 \pm 3.74$ & 4.94 & $1.2 \times 10^{-15}$ & \\
\hline $003743.1-334203$ & 03743.12 & -334203.96 & $72.32 \pm 8.72$ & 23.30 & $6.9 \times 10^{-15}$ & N.3, G1 \\
\hline $003743.3-334912$ & 03743.32 & -334912.53 & $224.16 \pm 16.79$ & 25.90 & $2.1 \times 10^{-14}$ & \\
\hline $003743.3-334312$ & 03743.35 & -334312.62 & $6.82 \pm 2.83$ & 2.86 & $6.5 \times 10^{-16}$ & N.18 \\
\hline $003743.6-334147$ & 03743.69 & -334147.24 & $14.43 \pm 4.00$ & 5.71 & $1.4 \times 10^{-15}$ & \\
\hline $003743.7-334534$ & 03743.76 & -334534.41 & $22.94 \pm 5.00$ & 8.58 & $2.2 \times 10^{-15}$ & \\
\hline 003743.8-334209 & 03743.85 & -334209.63 & $39.87 \pm 6.56$ & 13.42 & $3.8 \times 10^{-15}$ & N.2, G1 \\
\hline $003744.0-334028$ & 03744.00 & -334028.72 & $52.58 \pm 7.42$ & 18.90 & $5.0 \times 10^{-15}$ & \\
\hline $003745.2-334228$ & 03745.29 & -334228.31 & $73.21 \pm 8.77$ & 23.38 & $7.0 \times 10^{-15}$ & N.1, near G2 \\
\hline $003745.6-334151$ & 03745.61 & -334151.85 & $233.81 \pm 15.39$ & 78.32 & $2.2 \times 10^{-14}$ & \\
\hline $003745.7-334546$ & 03745.77 & -334546.64 & $31.46 \pm 5.83$ & 11.18 & $3.0 \times 10^{-15}$ & \\
\hline $003747.0-333952$ & 03747.07 & -333952.20 & $9.61 \pm 3.32$ & 3.90 & $9.2 \times 10^{-16}$ & G3 \\
\hline $003747.4-334104^{*}$ & 03747.44 & -334104.16 & $958.12 \pm 31.08$ & 243.33 & $9.2 \times 10^{-14}$ & \\
\hline 003748.6-333841 & 03748.67 & -333841.63 & $3.09 \pm 2.00$ & 1.35 & $3.0 \times 10^{-16}$ & \\
\hline $003748.8-334438$ & 03748.80 & -334438.51 & $130.79 \pm 11.62$ & 40.51 & $1.2 \times 10^{-14}$ & \\
\hline $003749.2-334404$ & 03749.20 & -334404.71 & $367.52 \pm 19.31$ & 105.15 & $3.5 \times 10^{-14}$ & \\
\hline $003749.3-334107$ & 03749.36 & -334107.58 & $85.18 \pm 9.38$ & 29.49 & $8.1 \times 10^{-15}$ & \\
\hline $003750.0-334641$ & 03750.01 & -334641.14 & $414.66 \pm 20.88$ & 72.74 & $4.0 \times 10^{-14}$ & \\
\hline $003751.2-334659$ & 03751.23 & -334659.26 & $14.39 \pm 4.24$ & 4.66 & $1.4 \times 10^{-15}$ & \\
\hline $003751.2-334216$ & 03751.25 & -334216.52 & $12.45 \pm 3.74$ & 4.95 & $1.2 \times 10^{-15}$ & \\
\hline 003751.4-334135 & 03751.41 & -334135.05 & $28.84 \pm 5.57$ & 10.65 & $2.8 \times 10^{-15}$ & \\
\hline 003751.6-334100 & 03751.65 & -334100.24 & $109.36 \pm 10.68$ & 32.91 & $1.0 \times 10^{-14}$ & \\
\hline $003752.4-334403$ & 03752.44 & -334403.45 & $155.06 \pm 12.65$ & 45.80 & $1.5 \times 10^{-14}$ & \\
\hline $003753.1-334131$ & 03753.16 & -334131.97 & $14.37 \pm 4.00$ & 5.65 & $1.4 \times 10^{-15}$ & \\
\hline $003754.1-334630$ & 03754.16 & -334630.93 & $64.90 \pm 8.66$ & 15.12 & $6.2 \times 10^{-15}$ & \\
\hline $003754.4-334630$ & 03754.43 & -334630.75 & $56.47 \pm 7.94$ & 15.27 & $5.4 \times 10^{-15}$ & \\
\hline $003754.5-334442$ & 03754.54 & -334442.30 & $13.65 \pm 4.36$ & 3.93 & $1.3 \times 10^{-15}$ & \\
\hline $003755.2-334159$ & 03755.28 & -334159.45 & $16.14 \pm 4.24$ & 6.17 & $1.5 \times 10^{-15}$ & \\
\hline $003755.7-334412$ & 03755.71 & -334412.00 & $21.81 \pm 5.20$ & 6.34 & $2.1 \times 10^{-15}$ & \\
\hline $003755.7-334755$ & 03755.75 & -334755.05 & $8.09 \pm 4.00$ & 2.05 & $7.7 \times 10^{-16}$ & \\
\hline $003756.3-334224^{*}$ & 03756.32 & -334224.76 & $992.62 \pm 31.72$ & 208.57 & $9.5 \times 10^{-14}$ & \\
\hline $003756.0-334221$ & 03756.05 & -334221.63 & $17.04 \pm 4.36$ & 6.44 & $1.6 \times 10^{-15}$ & \\
\hline 003801.2-334430 & 03801.26 & -334430.16 & $26.32 \pm 5.74$ & 7.06 & $2.5 \times 10^{-15}$ & \\
\hline
\end{tabular}

